\title{
Crisis Intervention Team Training and the Protection Motivation Theory
}

\author{
Monique Allen \\ Walden University \\ Gregory Campbell \\ Walden University
}

\begin{abstract}
The problem addressed in this phenomenological study was the lack of documentation that supported the lived experiences of crisis intervention team (CIT)-trained police officers related to their field encounters with persons with mental illnesses. The purpose of the study was to explore the lived experiences of officers among CIT-trained police officers who respond to persons with mental illnesses in field encounters to address the problem. The protection motivation theory was aligned closest with the teachings of CIT training as described by the study participants' lived experiences. Participants provided the study's collected data, which was composed of completed questionnaires and transcribed interviews. The empirical theoretical framework method of analysis used was a combination of inductive coding and theme analysis that established the results of this study. Key findings of the study identified a significant amount of frustration expressed in the lived experiences of the CIT-trained police officers. Frustration was experienced by officers who applied the protection motivation theory to ensure the well-being of persons experiencing a mental crisis. There was considerable pushback from the public mental health facilities, which added to the frustration experienced by CIT-trained police officers who attempted to navigate treatment with the limited resources available to help persons in mental crisis. The positive social change produced from this study included recommendations to police leadership and mental health advocates to encourage certain CIT-training-related practices that directly impact CIT field encounters with persons in mental crises. Specialized training may promote improved departmental outcomes such as sustainability of gains for those in crises and enable police officer accountability and reliability.
\end{abstract}

Keywords: crisis, mental health, intervention, sustainability of gains, CIT training

\section{Introduction}

\section{Problem Identification}

There are an estimated 3.9 million Americans with severe mental illnesses who receive no psychiatric treatment, and approximately $10 \%$ of all police contact with the public involves persons with mental illnesses (PwMI; Brown, 2015; Smith, 2012). Therefore, there is an increased chance of PwMI encounters that require crisis intervention team (CIT) training during the police's daily duties. The conceptual framework of this study included the shared lived experiences of CIT-trained officers concerning CIT training. Therefore, the problem addressed in this phenomenological study

This manuscript was completed in partial fulfillment of the requirements in Criminal Justice Program-Emergency Management Walden University, Minneapolis MN under the direction of Gregory Campbell, PhD. 
was the lack of documentation that supported the lived shared experiences of CIT-trained police officers related to their encounters with PwMI.

\section{Crisis Intervention Team Training}

In 1988, the CIT training model originated in Memphis, Tennessee, after a person with a mental illness was killed by a police officer. The model consisted of $40 \mathrm{hr}$ of CIT training over 5 days, and officers received CIT lapel pins at the end of the training (Dupont \& Cochran, 2007). CIT has led to decreased injuries to PwMI, increased transport to community mental health providers, and reduced jail suicides (Brown, 2015). CIT training entails teaching police officers verbal de-escalation skills that help eliminate agitation and decrease injuries during CIT field encounters. Browning, Van Hasselt, Tucker, and Vecchi (2011) itemized that the training curriculum included recognition and understanding of the signs and symptoms of mental illnesses, pharmacological interventions with possible side effects, crisis intervention and de-escalation skills, and knowledge of user-friendly mental health resources available to persons in mental crisis during CIT encounters. The training also provides officers with resources such as referrals to mental health care providers for long-term treatment as alternatives to incarceration. Most importantly, CIT training gives officers the ability to establish a connection with persons in crisis that may lead to a reduction in suicides. A new protocol was in order and it involved mental illness education, verbal de-escalation, collaboration with the mental health network, and transportation to mental health facilities instead of jail. There are many studies that have examined and explored CIT training for police officers since its inception approximately 30 years ago. Therefore, the purpose of the study was to explore the lived experiences of CIT-trained police officers to address the problem. This study explored the impact CIT training has on CIT-trained officers and found out whether improved perceptions come from CIT training or more so from the support of the mental health clinicians and available mental health facilities. This study could have significant policy implications for tracking and monitoring referrals and treatment from CIT encounters. By exploring the lived experiences of CIT officers, this study revealed how the protection motivation theory (PMT) was pivotal to the success of CIT encounters.

\section{Protection Motivation Theory and CIT}

The biggest shift in the paradigm of treatment for PwMI in crisis was toward a method of protection. In 1975, Rogers introduced the PMT, which researchers primarily use as the framework for intervention in health-related behaviors to measure the correlation between threat and coping appraisal variables (Milne, Sheeran, \& Orbell, 2000). Milne et al. opined that the basis of Rogers's PMT was fear appeal, with countermeasures to reduce the impact of fear with healthy interventions. The PMT continues to exhibit evolved and more polished officer perceptions and encompasses new strategies that strengthen mental-health-related network behaviors. Thus, instead of fearing persons in mental crisis, officers can apply the PMT to refer to such persons with care and give soft diagnoses in CIT encounters. Because of this, CIT training reduces the fear of making a PMT diagnosis in the field.

Milne et al. (2000) identified that a strategic approach to eliminating fear arousal begins with a threat appraisal in which the individual's perceptions of a threat and perceived vulnerability demonstrate how susceptible the person feels to the communicated threats. Milne et al. also noted that Rogers revised the PMT model in 1983 to encompass a reward element for committing to a change in behavior or habit, in which the higher the reward, the less likely the individual is to retreat to old habits. Therefore, successful intervention becomes a personal reward for CIT-trained 
officers who help persons in mental crisis reach a sustainability of gains, where CIT interventions are no longer required because the person successfully achieved mental stability.

The sustainability of gains is reached when mental health is restored to normal capacity from the point of crisis, which typically happens after the first year of treatment, and the "sweet spot" of mental stability has been achieved when PwMI no longer require crisis monitoring by CIT-trained officers (B. Stettin, personal communication, August 24, 2017). To this end, emergency psychiatric first responders such as law enforcement agents and mobile crisis teams began a new movement to implement nationwide CIT training. A close alignment exists between the PMT and the dynamics of CIT training for police officers. Brown (2015) noted that mobile crisis teams are interdisciplinary teams of mental health professionals, such as social workers, nurses, psychiatrists, psychologists, addiction specialists, and peer counselors. Together, the CIT and mobile crisis team units respond to persons in mental crisis and use any criminal diversion programs available to help stabilize individual mental capacity.

\section{Research Design}

The objective of the phenomenological research design method was to obtain thorough descriptions of shared lived experiences that depict the basis for reflective structural analysis (Moustakas, 1994; Ravitch \& Carl, 2016). The Walden University Institutional Review Board granted permission to conduct this research (approval no. \#03-07-18-0668953). The qualitative interviews in this study were suitable for exploring the impact of CIT training on CIT-trained officers, which revealed both positive and negative experiences. Rubin and Rubin (2012) documented that researchers who conduct semistructured interviews discuss how events took place as an extended conversation between researcher and interviewee, with limited questions and follow-up questions. Accordingly, this study used a questionnaire and also conducted semistructured interviews with follow-up questions that allowed participants to contribute to the body of knowledge about CIT training lived experiences.

\section{Methodology}

The alignment from the study's research topic to the research question on how officers' perceptions improved based on CIT training when encountering PwMI to the methodology and to the design was consistent. When researchers collect qualitative data, the intention is to explore the topic with selected participants at sites (Creswell and Creswell, 2017). To this end, the research unit of analysis was CIT-trained officers. Thus, the purposive random sample consisted of seven out of 20 possible CIT-trained police officers in the precinct. Because of this, the purpose of the phenomenological study was to explore the impact of CIT training for CIT-trained police officers. The study's strength originated from the participant pool with a focus solely on police officers in one region.

Research Question: How do officers' perceptions of PwMI improve with CIT training?

\section{Data Collection}

The data collection site was predetermined by the police department's sergeant in one of three divisions of Contra Costa County, California. Participants included a random selection of CIT officers (identified only by their initials) who were available that week to interview during the day shift. Invitations to participate in the study were distributed to the randomly selected participants via email by the department's lieutenant. On the days of the interviews, confidential consent forms were 
distributed to each participant for signature prior to initiating the in-depth interview process. Questionnaire distribution was followed by the interviews. This instrument was developed by a project team encompassing an eclectic group of stakeholders: police officials, social workers, judicial representatives, mental health service providers, PwMI, and family members of such persons (Wells \& Schafer, 2006). Interviews were scheduled for an hour, and all participants in the precinct were interviewed within the same week. Two recording devices were used to collect the phenomenological data from the seven participants to capture clarification requests while filling out the questionnaire as well as during the open-ended questions.

\section{Data Analysis}

The paper questionnaires were counted and analyzed manually and entered into Microsoft Excel as frequency tallies and percentages calculated from the participants' confidential responses.

Approximately $4.5 \mathrm{hr}$ of recorded audio interviews were transcribed and used for data analysis. The researcher transcribed the interviews using the intelligent technique that eliminated pauses in the participant interviews. The interview audio files were transcribed verbatim by a third party to maintain data integrity and validity. The transcribed data was coded to detect themes and the NVivo 12 software application determined emerged theme validation.

\section{Findings}

CIT-trained officers appreciated the specialty training once trained, not before. Data analysis revealed officers who had healthy positive attitudes about persons in crisis and yet were despondent with the lack of resources and support available to help people in mental crisis. Participants shared lived experiences included mostly negative sentiments from the frustration theme and mostly positive sentiments from the teachings of CIT training. Officers took the time required to establish the connection with the person in mental crisis. There was a notable frustration theme analyzed by direct quotes from the in-depth interviews. Frustration resulted from the lack of resources and support from nonlocal resources along with substantial pushback. There is a significant lack of local mental health facilities with inpatient psychiatric beds for long-term treatment. Local therapists or case workers who are available to respond to mental crises that happen after business hours or on weekends rarely exist. Pushback involves homeless persons in mental crisis who do not want to leave their belongings for treatment. The jails give tickets for misdemeanor offenses to those in crises instead of adequate mental health treatment. The study explored CIT-trained officer perceptions of CIT training and revealed an improvement with a sense of caring and compassion. Officers' improved perceptions came from either CIT training or being raised in an accepting and tolerant household. Further, the study's research question was answered, CIT training improved perceptions from officers who did not already have compassion for PwMI. Moreover, mental health clinicians educating CIT officers also improved officers' perceptions of PwMI crisis. Table 1 indicates that 71\% of patrolmen indicated five or more CIT field encounters per month. 
Table 1. Officers' Perceptions of Crisis Intervention Team Field Encounters

\begin{tabular}{lcc}
\hline & $N$ & $\%$ \\
\hline $\begin{array}{l}\text { On average, how many contacts do you have } \\
\text { per month with persons in crisis? }\end{array}$ & \\
$<1$ & 1 & 14.5 \\
$1-2$ & 1 & 14.5 \\
$3-4$ & 0 & 0 \\
$\geq 5$ & 5 & 71 \\
\hline
\end{tabular}

Table 2 shows that the majority (56\%) of CIT-trained officers spend 30-60 min with CIT encounters, while $44 \%$ of CIT-trained officers spend fewer than $30 \mathrm{~min}$. All CIT-trained officers (100\%) said they believe ensuring the safety of people involved is very important. When asked about the importance of ensuring persons in mental crisis get mental health services, $56 \%$ of CIT-trained officers said it is very important, and $44 \%$ said it is somewhat important.

Table 2. Officers' Perceptions of Crisis Intervention Team Encounter Outcomes

\begin{tabular}{lcc}
\hline & $\boldsymbol{N}$ & $\mathbf{\%}$ \\
\hline Average time spent in contact with persons in crisis & & \\
$\quad<30 \mathrm{~min}$ & 3 & 44 \\
$\quad 30-60 \mathrm{~min}$ & 4 & 56 \\
Ensuring the safety of people involved & & 100 \\
$\quad$ Very important & 7 & \\
$\quad \begin{array}{l}\text { Ensure persons in crisis received mental health } \\
\text { services }\end{array}$ & & 56 \\
$\quad$ Very important & 4 & 44 \\
$\quad$ Somewhat important & 3 & \\
\hline
\end{tabular}

Additionally, the majority of officers (56\%) said they would appreciate an easier way to get PwMI to treatment facilities. All officers were dissatisfied with the process of placing persons in mental crisis in a mental health facility ( $72 \%$ said they were very dissatisfied and $28 \%$ somewhat dissatisfied). The frustration about current limited, nonlocal resources was apparent: $44 \%$ of officers agreed and $56 \%$ disagreed that there should be a new place for evaluation in the area. The disjoint in response stems from the feeling of hopelessness experienced by officers who attempt to get sustainable help for persons in mental crises. There were conflicting sentiments CIT-trained officers expressed via the questionnaire collected data. There was an overall positive appreciation for the specialty training; however, the negative sentiments reflected the lack of resources and support CIT-trained officers had during CIT field encounter outcomes.

\section{Discussion}

One implication of social change of this study is that tracking CIT-related field encounters to can help with officer procedures and improved responses. Another implication is that CIT training can provide reliable mental health care. This study may also validate the importance of and bring value to CIT training for police officers and promote its standardization in each county where the collaboration between law enforcement and the mental health networks represents a united front of support for PwMI. That said, this study's recommendations provide suggestions for the treatment of PwMI in crisis. 


\section{Conclusions}

After interviewing the population of Contra Costa County's CIT-trained officers, the data analysis showed that the department in one of the socioeconomically challenged county divisions was severely depleted of resources and support for PwMI in crisis. There was a great disconnect at the county level of operations. It appears that fiscal needs are met when a county's affluent areas receive more resources and support than the socioeconomically challenged areas. One area of Contra Costa County represents the lower economic bend of the county. The central division has ample resources for its affluent neighborhoods, and the other county divisions have similar affluent residents with access to central resources through joint partnership taskforces. However, one division will remain neglected without the implementation of one vital new legislative bill that could aid the entire Contra Costa County with its current homeless PwMI population-A.B. 1971 (2018).

\section{Recommendations for Action}

A.B. 1971 (2018) stated that facilities will have 8- to 24-hr detention and 72-hr hold capabilities. New local resources and support will provide substantial relief to the constant pushback from the distant psychiatric facilities with limited inpatient capacity. Conservatorship for the gravely disabled is for 1 year, and PwMI who qualify for an appointed guardian can petition for an additional year for (A.B 1971, 2018). A.B. 1971 stated that appropriate placement is in a licensed psychiatric health care facility, community-based residential care setting, or supportive housing that provides wraparound services.

Contra Costa County ambulatory care requests are fulfilled by the American Medical Response ambulances that get pulled out of rotation from over 30 miles away, during noncommute hours, to respond to all county CIT encounters. Each division of Contra Costa County needs the ability to physically transport consumers to various treatment facilities using its own CIT vehicles that protect the identity of PwMI. Currently, pairs of officers respond to 5150 calls (i.e., calls for evaluation of a patient being held at a psychiatric facility) without assistance of clinicians. Lastly, we suggested that the mental health evaluation team be staffed to correspond in pairs with CIT-trained officers and provide CIT training to officers in each Contra Costa County division it represents.

\section{Connections}

A close alignment exists between the PMT and the dynamics of CIT training for police officers. This study established the answer to the study's research question concerning CIT-trained officers' perceptions of CIT training. CIT-trained officers embraced the specialty training and practiced its teachings during CIT encounters. In conclusion, the officers from every Contra Costa County require field support from each county division of the mental health evaluation team by providing coresponder pairings with CIT-trained patrolmen.

\section{References}

Brown, R. L. (2015). Decriminalizing mental illness: The need for treatment over incarceration before prisons become the new asylums for the mentally ill. Psychology Summer Fellows. 1. Retrieved from http://digitalcommons.ursinus.edu/psych_sum/1

Browning, S., Van Hasselt, V., Tucker, A., \& Vecchi, G. (2011). Dealing with individuals who have mental illnesses: The crisis intervention team (CIT) in law enforcement. British Journal of Forensic Practice, 13, 235-243. doi:10.1108/14636641111189990 
Creswell, J. W., \& Creswell, J. D. (2017). Research design: Qualitative, quantitative, and mixed methods approaches. Thousand Oaks, CA: Sage.

Dupont, R., \& Cochran, S. (2007). Police response to mental health emergencies: Barriers to change. The Journal of the American Academy of Psychiatry and the Law, 28, 338-344.

Mental health services: Involuntary detention: gravely disabled, AB 1971. (2018). Retrieved from https://leginfo.legislature.ca.gov/faces/billTextClient.xhtml?bill_id=201720180AB1971

Milne, S., Sheeran, P., \& Orbell, S. (2000). Prediction and intervention in health-related behavior: A meta-analytic review of protection motivation theory. Journal of Applied Social Psychology, 30, 106-143.

Moustakas, C. E. (1994). Phenomenological research methods. Thousand Oaks, CA: Sage.

Ravitch, S. M., \& Carl, N. M. (2016). Qualitative research: Bridging the conceptual, theoretical, and methodological. Thousand Oaks, CA: Sage.

Rubin, H. J., \& Rubin, I. S. (2012). Qualitative interviewing: The art of hearing data (3rd ed.). Thousand Oaks, CA: Sage.

Smith, C. (2012). Neoliberalism and individualism: Ego leads to interpersonal violence. Sociology Lens. Retrieved from https://www.sociologylens.net/topics/crime-and-deviance/neoliberalismindividualism-ego-violence/11193

Wells, W., \& Schafer, J. A. (2006). Officer perceptions of police responses to persons with a mental illness. Policing, 29, 578-601.

The Journal of Social Change, sponsored by Walden University, welcomes manuscripts focusing on interdisciplinary research in social change that improves the human condition and moves people, groups, organizations, cultures, and society toward a more positive future.

Walden University Publishing: http://www.publishing.waldenu.edu 